\title{
Minimizing the Cost in Sample Size Computation
}

\author{
J iangao Luo* \\ Department of Biostatistics, University of Nebraska \\ Medical Center, USA \\ *Corresponding author: Jiangao Luo, Department \\ of Biostatistics, College of Public Health, University of \\ Nebraska Medical Center, 984375 Nebraska Medicine, \\ Emile and $42^{\text {nd }}$ St, Omaha, NE 68198-4375, USA
}

Received: November 16, 2016; Accepted: November 21, 2016; Published: November 22, 2016

\section{Editorial}

While our society is hungry for new medical treatments that can bring hopes to millions of patients at the terminal of their lives, the medical experiment expenses are sky rocketing, funding sources for research are limited and shrinking. It is even worse that our research budgets for awarded proposals are often cut to a fraction of original planned spending. Therefore, it is very important for us to find methods for minimizing costs in medical studies. In 1977, Cochran [1] studied how to minimize the cost for survey samplings in his classic book. Ideal solution in sample size allocation is to maximize the power and minimize the cost with required significant level. Guo et al. [2] have some nice results in this area. Allison et al. [3] have discussed strategies in minimizing the financial cost while minimizing the cost, but their paper lacks depth in computing. Guo and Luh [4] have studied how to compute sample size with fixed cost for comparing two trimmed means. Luo, Wang and Meza [5] have proven the formulas of sample size with maximal precision for difference and ratio of two binary data and maximal power for detecting the difference of two proportions, two survival rates and two correlations under financial constraints. The free software for their results is available upon request. There are a lot of publications about cost-effectiveness-design (see [6,7] and accompanying references), but our approaches are totally different from them since we focus on sample size allocation at the design stage. Our methods are statistics with computational support.

As we conduct sample size computation for clinical trials with cost in mind, there are many questions for us to work on and much room for improvement. Since clinical trials are experiments on humans, we will face unpredictable things and corresponding costs are in some sense unpredictable. But there are still some rules that we can follow and a lot of things are within the control of our ability. That is why we have budget sheet in our research proposal. There are items for the cost of clinical trials that may be listed, say nurses, technicians, physicians, managers, medicines, compensation, insurance, tests, lab, etc., but we can classify them as two parts, one that is fixed cost, and the other will be proportional to the sample sizes of control and intervention groups. Let us assume the sample sizes for intervention and control are $N_{1}$ and $N_{2}$, respectively, in a clinical trial and corresponding costs for each patient are $C_{1}$ and $C_{2}$, respectively, then our interest is to minimize the costs from the second part at the design stage. The objective functions for minimization are the cost functions related to sample sizes $N_{1}$ and $N_{2}$, prices $C_{1}$ and $C_{2}$, power, significant level, variances, and minimal detectable effect size. For example, if our interest is non-inferiority or superiority test for two proportions with the following hypotheses

$$
H_{0}: \pi_{1}-\pi_{2} \leq \Delta v s H_{1}: \pi_{1}-\pi_{2}>\Delta
$$

Where $\pi_{1}$ and $\pi_{2}$ are the response rates in intervention and control, then the optimal sample allocation that minimizing the total cost is [8]

$$
\begin{aligned}
& N_{1}=\frac{\left[\pi_{1}\left(1-\pi_{1}\right)\right]^{\frac{1}{2}}\left(\left[C_{1} \pi_{1}\left(1-\pi_{1}\right)\right]^{\frac{1}{2}}+\left[C_{2} \pi_{2}\left(1-\pi_{2}\right)\right]^{\frac{1}{2}}\right)}{C_{1}^{\frac{1}{2}}\left(\frac{\pi_{1}-\pi_{2}}{z_{\frac{\alpha}{2}}+z_{\beta}}\right)^{2}} \\
& N_{2}=\frac{\left[\pi_{2}\left(1-\pi_{2}\right)\right]^{\frac{1}{2}}\left(\left[C_{1} \pi_{1}\left(1-\pi_{1}\right)\right]^{\frac{1}{2}}+\left[C_{2} \pi_{2}\left(1-\pi_{2}\right)\right]^{\frac{1}{2}}\right)}{C_{2}^{\frac{1}{2}}\left(\frac{\pi_{1}-\pi_{2}}{z_{\frac{\alpha}{2}}+z_{\beta}}\right)^{2}}
\end{aligned}
$$

Note we do not have equal sample size allocation. Here equal sample size allocation does not save the budget. Luo et al. [8-10] have obtained a series of results in this area with required power and significant level under different conditions. Please see our follow-up papers for details. Another area that is to follow is what Luo, Wang, and Meza [5] have done and to work out results under financial constraints. Assume our given total cost under the above hypothesis is $C$ and we want to test the difference of two independent sample means with normal distributions, then for the following hypotheses

$$
H_{0}: \mu_{1}=\pi_{2} v s H_{1}: \mu_{1} \neq \mu_{2}
$$

the following sample size allocation reaches maximal power [5]

$$
N_{1}=\frac{C \sigma_{1}}{\sqrt{C_{1}}\left(\sigma_{1} \sqrt{C_{1}}+\sigma_{2} \sqrt{C_{2}}\right)}, N_{2}=\frac{C \sigma_{2}}{\sqrt{C_{2}}\left(\sigma_{1} \sqrt{C_{1}}+\sigma_{2} \sqrt{C_{2}}\right)}
$$

We deeply believe the results are useful and will be more efficient economically in the design of medical studies.

We also develop software available for all users for free. This is very important for the wide application of a statistical method that will be beneficial to all clinical trials. Our software will be published and available to public users. Software does not only test our methods, but also provides feedbacks for further development.

\section{References}

1. Cochran W. Sampling Techniques, $3^{\text {rd }}$ ed., Wiley, New York. 1977; 448.

2. Guo JH, Chen $\mathrm{HJ}$, Luh WH. Sample size planning with the cost constraint for testing superiority and equivalence of two independent groups. British Journal of Mathematical and Statistical Psychology. 2011; 64: 439-461.

3. Allison DB, Allison RL, Faith MS, Paultre F, Pi-Sunyer FX. Power and money: Designing statistically powerful studies while minimizing financial costs, Psychological methods. 1997; 2: 20-33.

4. Guo JH, Luh WH. Optimum sample size allocation to minimize cost or maximize power for the two-sample trimmed mean test. British Journal of 
Mathematical and Statistical Psychology. 2009; 62: 283-298.

5. Luo J, Wang Y, Meza J. Optimal sample size allocation under financial constraint. Austin Biom and Biostat. 2015; 2: 1024

6. Ramsey S, Willke R, Briggs A, Brown R, Buxton M, Chawla A, et al. Good research practices for cost-effectiveness analysis alongside clinical trials: The ISPOR RCT-CEA Task force report. Value in Health. 2005; 8: 521-533.

7. Ramsey S, Willke R, Briggs A, Glick H, Reed S, Augustovski F, et al. Cost effectiveness analysis alongside clinical trials II - An ISPOR Good research practices task force report. Value in Health. 2015; 18: 161-172.
8. Luo $\mathrm{J}$, Lin $\mathrm{Z}$, Wang $\mathrm{Y}$, Wang $\mathrm{H}$, Luo $\mathrm{B}$. Minimizing the cost in sample size calculation of comparing two proportions (to appear).

9. Luo J, et al. Optimal sample size allocation in testing relative risk of two proportions (to appear).

10. Luo $\mathrm{J}$, et al. Minimizing the cost in sample size allocation in comparing two means (to appear).
Austin Biom and Biostat - Volume 3 Issue 1 - 2016

Submit your Manuscript | www.austinpublishing group.com

Luo. (C) All rights are reserved
Citation: Luo J. Minimizing the Cost in Sample Size Computation. Austin Biom and Biostat. 2016; 3(1): 1031. 